\title{
Role of Endoscopic Gastroplasty Techniques in the Management of Obesity
}

\author{
Yunho Jung \\ Division of Gastroenterology, Department of Internal Medicine, Soon Chun Hyang University College of Medicine, Cheonan, Korea
}

Health and wellness represent a major global concern. Trends such as a lack of exercise and excessive consumption of calories are major causes of the rapid increase in obesity worldwide. Obesity should be controlled because it can result in other illnesses, such as diabetes, high blood pressure, high cholesterol, coronary artery disease, stroke, breathing disorders, or cancer. However, many people have difficulty in managing obesity through exercise, dietary control, behavioral modifications, and drug therapy. Bariatric surgery is not commonly used due to a variety of complications, even though it has been demonstrated to produce reliable results with respect to adequate weight loss when performed using an open or a laparoscopic approach. Endoscopic bariatric procedures are emerging techniques that are less invasive and safer compared with current surgical approaches. However, the evaluation of endoluminal procedures is limited by the small number of studies and their short-term follow-up. Clin Endosc 2017;50:21-25

Key Words: Bariatric surgery; Obesity; Weight loss

\section{INTRODUCTION}

Obesity has emerged as a major health threat in Korea, as well as globally, in recent years. According to Korea Health Statistics 2011, obesity affects one-third of Korean adults. ${ }^{1}$ The importance of obesity should be emphasized because it is a major risk factor for cardiovascular disease, hypertension, and diabetes mellitus. ${ }^{2}$ Obesity also increases the risk of comorbidities, such as gastroesophageal reflux disease (GERD), biliary disease, non-alcoholic fatty liver disease, non-alcoholic steatohepatitis, and gastrointestinal (GI) cancer. ${ }^{3}$ However, the majority of obese patients fail to achieve sustained weight reduction or optimal weight by reduced calorie intake, a low-fat diet, exercise, or some combination thereof. If weight loss is

Received: November 3, 2016 Revised: December 4, 2016

Accepted: December 13, 2016

Correspondence: Yunho Jung

Division of Gastroenterology, Department of Internal Medicine, Soon Chun Hyang University College of Medicine, 31 Suncheonhyang 6-gil, Dongnam-gu, Cheonan 31151, Korea

Tel: +82-41-570-3741, Fax: +82-041-574-5762, E-mail: c73138@schmc.ac.kr

(cc) This is an Open Access article distributed under the terms of the Creative Commons Attribution Non-Commercial License (http://creativecommons.org/ licenses/by-nc/3.0) which permits unrestricted non-commercial use, distribution, and reproduction in any medium, provided the original work is properly cited. achieved, it is usually difficult to reduce more than $10 \%$ of total body weight and sustain the effects for a prolonged period.

Bariatric surgery has shown good results in achieving adequate and reliable weight loss using either a laparoscopic approach or an open technique. The number of bariatric surgeries performed in the US has continued to increase annually, from 158,000 procedures in 2011 to 193,000 procedures in $2014 .^{4}$

Bariatric surgery is not a straightforward procedure, although it has known benefits, such as long-term weight loss and improved obesity-related comorbidities. This is in part because it may result in complications that can be life threatening. Furthermore, postoperative GI and surgical complications can occur with different types of surgery, including anastomotic leaks, fistulas, stricture, GI bleeding, staple line disruption, bezoars, internal hernia, peritonitis, and band slippage, or migration. ${ }^{5}$ Risk stratification may prevent many of these patients from gaining access to surgical interventions for weight loss. Endoscopic approaches are similar to surgical methods, although they are less invasive, safer, and more cost-effective than current surgical approaches. However, the evaluation of endoscopic bariatric procedures is limited by the small number of published studies and sample size, and 
the long-term effects are currently not well known. Finally, endoscopic restriction procedures have not shown additional remarkable benefits beyond surgery until now.

Thus, this article reviewed studies describing endoscopic gastroplasty and discussed its role in the management of obesity.

\section{GASTROPLASTY AND SURGICAL GASTROPLASTY}

Gastroplasty is a type of weight loss surgery to treat morbid obesity. It is a restrictive procedure in that it reduces the gastric cavity and limits the amount of food intake, whereas alternative procedures, such as gastric bypass, work by reducing the absorption of nutrients and calories in the digestive tract to limit caloric intake. Following gastroplasty, satiety can be achieved following the intake of a small amount of food due to a reduction in the size of the stomach cavity, thereby facilitating reduction in body weight. Additionally, gastroplasty does not cause nutritional deficiencies, including anemia, which occurs following gastric bypass because it does not involve alterations in the anatomy of the GI tract. However, the ingestion of high-caloric solid foods and fluids eventually occurs in most of these patients, which results in weight gain and recurrence of the associated comorbid conditions. ${ }^{6}$ Strict follow-up and regular monitoring with well-planned dietary intake and exercise are beneficial to maintaining weight loss after gastroplasty.

In recent decades, various bariatric procedures have been performed, including vertical-banded gastroplasty (VBG), Roux-en-Y gastric bypass, biliopancreatic diversion, laparoscopic-adjustable gastric banding, and laparoscopic sleeve gastrectomy. $\mathrm{VBG}^{7}$ and silastic ring vertical gastroplasty (SRVG) ${ }^{8}$ are widely used gastroplasty procedures for managing obesity.

These procedures are performed by stapling the stomach in continuity and vertically, thereby leaving a 10-30 cc upper proximal stomach pouch. A small gap in the staple-line is left, which causes digested food in the pouch to slowly descend into the distal stomach. To reduce the possibility of preventing stretching and enlarging of the passage into the distal stomach, a tight plastic band or a silastic ring is placed around the upper stomach.

Currently, most surgeons who perform gastroplasties transect the stomach to decrease the incidence of staple-line breakdown. ${ }^{6}$ Laparoscopically, transection of the stomach is currently performed relatively easily when attempting various gastroplasties. The outcome of gastroplasties in prospective clinical evaluations yields an average percentage excess weight loss of approximately $50 \%$ in approximately $50 \%$ of patients. ${ }^{10}$
The advantages of gastroplasty operations include its simplicity, lack of a need to rerout the GI tract, reduced invasiveness, and no malabsorption. However, complications include vomiting, GERD, staple-line breakdown, band erosion, outlet stenosis, and obstruction. This is in addition to complications that are common to all other GI operations, such as wound infection, bleeding, incisional hernias, leaks, fistula formation, deep vein thrombosis, and pulmonary embolism. ${ }^{6}$

\section{ENDOSCOPIC BARIATRIC PROCEDURES AND ROLE}

Recent surgical trends have sought to achieve a smaller scar and use a minimally invasive approach. Endoluminal bariatric procedures, which are performed using flexible endoscopy completely through the GI tract, offer the potential for an ambulatory weight loss procedure that may be more cost-effective and safer than current surgical bariatric procedures. ${ }^{11}$ Endoscopic bariatric procedures have been attempted as a type of relatively noninvasive approach related to the restriction of food intake or malabsorption.

Several roles for endoscopy in the management of obesity have been suggested. For example, endoscopy can be used as an early intervention in patients with early stage obesity as a bridge to surgical procedures to reduce obesity-related operative risks; as a metabolic procedure that primarily addresses comorbid illnesses such as diabetes; as a primary obesity procedure that seeks outcomes similar to those of traditional bariatric surgery with reduced periprocedural risk; and, finally, as a revision procedure for failed bariatric surgical procedures. ${ }^{12}$ However, the evaluation of endoluminal endoscopic bariatric procedures is limited by the small number of patients enrolled and the short follow-up period therein.

\section{TYPES OF ENDOSCOPIC GASTROPLASTY}

\section{Transoral gastroplasty (with the TOGA system)}

The Transoral Gastroplasty (TOGA) system (Satiety Inc., Palo Alto, CA, USA) represents the first endoscopic device designed primarily to create a vertical gastric sleeve. The TOGA system has two disposable staplers: the TOGA Sleeve stapler and the TOGA Restrictor. The TOGA Sleeve Stapler is introduced over the guide wire after dilation with the Savary bougie. A smaller gastroscope is advanced through the device and retroflexed to directly visualize the stapler. The sleeve stapler is tightened to acquire a double-fold of tissue with a strong vacuum, and the staples are then fired. This process is repeated to add a second staple line. Subsequently, the sleeve outlet 
is constricted using the TOGA restrictor, which is acquired with a vacuum and the stapler. Additional restrictions are in place to reduce the sleeve outlet from approximately 20 to 10 $\mathrm{mm}$ in diameter. This allows completion of a restricted gastroplasty along the lesser curvature of the proximal stomach. ${ }^{13}$ Following the procedure, the amount of food or liquids that the patient can eat is limited by the size and anatomy of the gastric pouch, with an accompanying feeling of early satiety. ${ }^{14}$

The first human multicenter study using the TOGA system has been reported. Twenty patients with a mean body mass index (BMI) of 43.3 (range, 35-53) kg/ $\mathrm{m}^{2}$ were enrolled. Although device-related adverse events, such as vomiting, pain, nausea, and transient dysphagia, were reported, no serious adverse events occurred. The mean weight loss of patients was $16.2 \%$ at 1 month, $22.6 \%$ at 3 months, and $24.4 \%$ at 6 months. However, a concern with this pilot study was the high incidence (61.9\%; 13/21 patients) of gaps between the two staple lines at 6-months follow-up endoscopy. ${ }^{13}$

The technique was subsequently improved by the development of an adjustable septum, thereby allowing closer apposition of the two staple lines to reduce the gap between the staple lines and the progression of the ischemic process that is related to transmural stapling. In a second human pilot study, there were no serious adverse events, and the mean weight loss was $19.2 \%, 33.7 \%$, and $46.0 \%$ at 1,3 , and 6 months, respectively. The occurrence and size of the gap between the staple lines was reduced, but $36.3 \%$ (4/11) of the patients had a mid-stoma $(<1 \mathrm{~cm})$ visible endoscopically at 6 months. ${ }^{15}$

\section{Endoluminal vertical gastroplasty}

The EndoCinch" suturing system (C.R. Bard Inc., Murray Hill, NJ, USA) was originally designed for the endoluminal endoscopic treatment of GERD. ${ }^{16}$ This system includes a capsule that is attached at the tip of the endoscope, a knot pusher, a tag pusher, tags attached to sutures, and a suture cutter. ${ }^{17}$ Sutures were made by deploying the T-tag suture after sucking the mucosa and submucosa of the gastric wall into the capsule.

The first stitch is placed proximally in the nearest folds on the anterior face of the gastric fundus. Then, the stitch configuration comprises one continuous suture running through five to seven bites of tissue or stitch points. An illustration of a continuous stitch configuration is given in Fig. 1. After the stitches are placed and visualized, the suture is tightened, thereby bringing the anterior and posterior faces together to create the endoluminal vertical gastroplasty. ${ }^{18}$

Fogel et al. ${ }^{18}$ evaluated the feasibility and effectiveness of the EndoCinch suture system for weight loss in 64 patients (mean age $=31.5$ years). The mean procedure time was approximately 45 minutes. The patients experienced a significant reduction

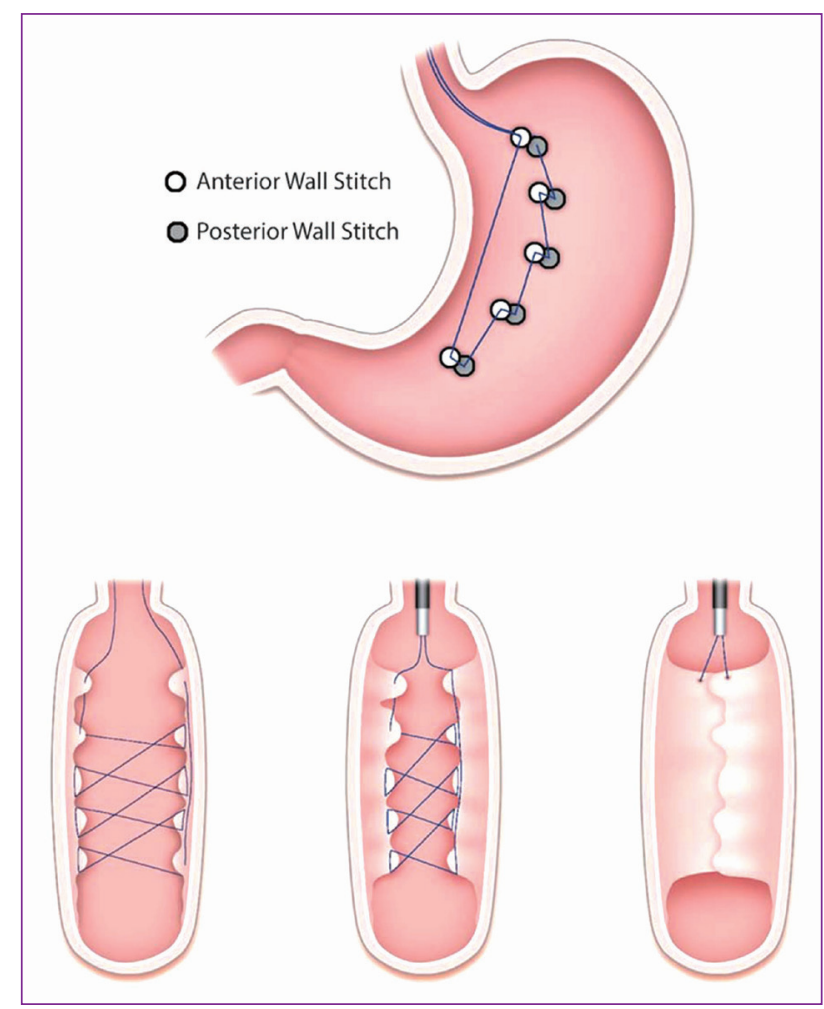

Fig. 1. An illustration of the endoluminal vertical gastroplasty stitching pattern with cross-section views. ${ }^{18}$

in BMI at 12 months (mean [SD] BMI $=39.9 \pm 5.1 \mathrm{~kg} / \mathrm{m}^{2}$ vs. $30.6 \pm 4.7 \mathrm{~kg} / \mathrm{m}^{2}, p<0.001$ ) and the amount of weight loss, relative to total weight, was $21 \%, 39.6 \%$, and $58.1 \%$, at 1,3 , and 12 months after the procedure, respectively. However, there was some concern regarding the durability of the plication, as only approximately $35 \%$ of the suture configurations remained fully intact during the follow-up period.

The Restore Suturing System (Bard Davol, Warwick, RI, USA) is a modified version of the previous EndoCinch Suturing System that overcomes the requirement for an overtube, multiple esophageal intubations, and a second endoscope to position the fastening device. This device allows multistitch suturing and suture fastening to be performed during a single intubation.

A study using this device assessed the weight reduction of 18 patients (mean age $=40$ years) after 12 months. The average number of plications placed per patient was six, and the mean procedure time was 2.1 hours. At the 12-month follow-up, 14 patients achieved statistically significant decreases in mean weight $(11.0 \mathrm{~kg})$ and mean BMI $\left(4.0 \mathrm{~kg} / \mathrm{m}^{2}\right){ }^{19}$

\section{POSE}

This procedure is performed using the Incisionless Operating Platform (IOP; USGI Medical, San Clemente, CA, USA). The device creates tissue plications by opposing tissue 


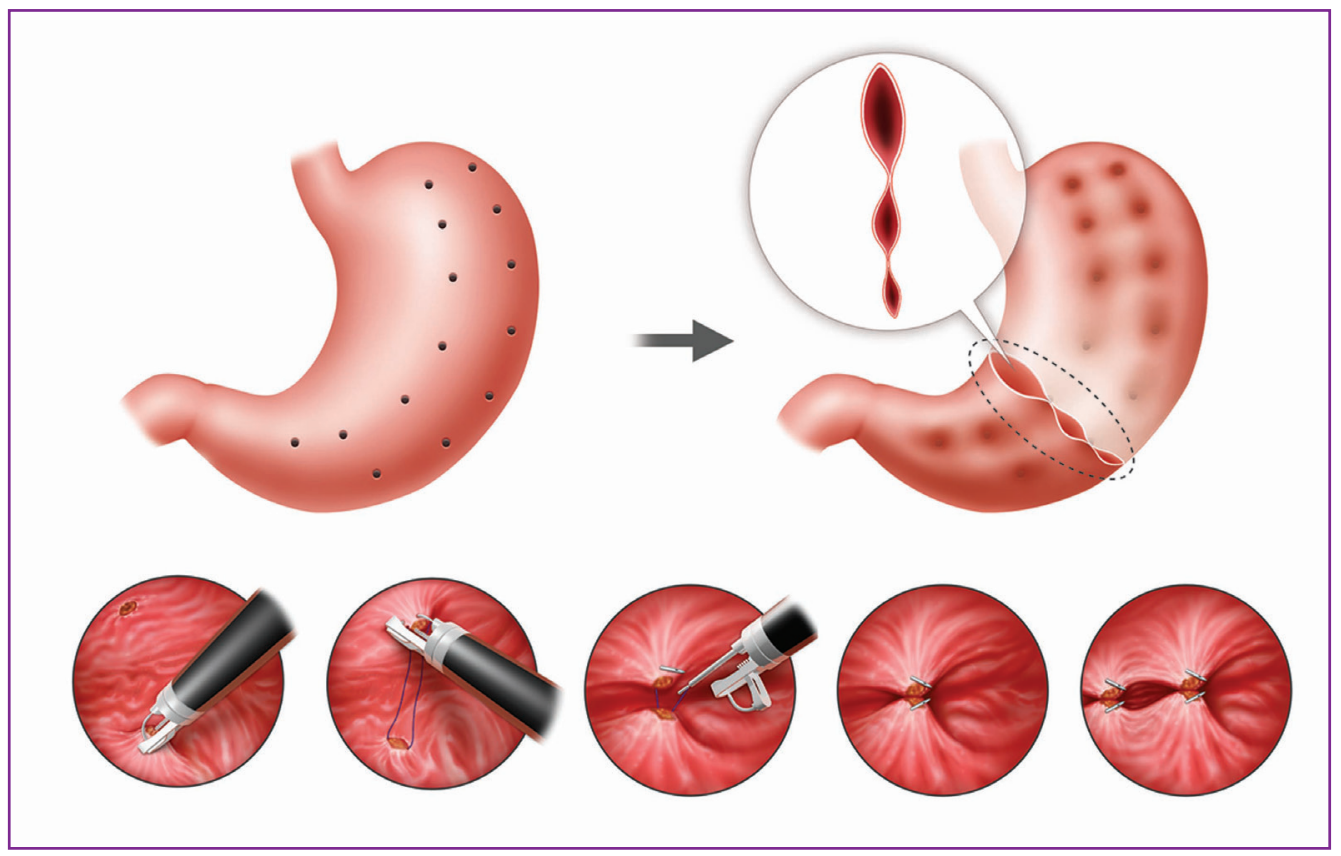

Fig. 2. An illustration of the suturing sequence for the creation of the endoscopic sleeve gastroplasty. ${ }^{21}$ and then deploying and anchoring full-thickness stitches. It uses a four-channel platform with a visualization endoscope, a rotatable tissue grasper, and suture cutter (g-Prox), a tissue helix (g-Lix), and a suture anchor deployment catheter (g-Cath). During the primary obesity surgery endoluminal (POSE) procedure, stomach tissue is plicated in eight or nine locations in the fundus and in three or four locations in the distal body, using specialized suture anchors. The plicated areas mechanically and physiologically restrict contact between the ingested food and the full surface area of the stomach.

A recent study reported the results of the POSE procedure in 147 patients (mean age $=43.8$ years). The mean procedure time was approximately 35 minutes and an average of 7.5 and 3.5 suture anchors were placed in the fundus of the stomach and distal body, respectively. The baseline weight was significantly reduced at 3,6 , and 12 months after the POSE procedure $(p<0.05)$. The patients experienced a mean total weight loss of $15 \mathrm{~kg}$ at 6 months, and the 116 patients who were available for follow-up at 1 year had a mean total weight loss of $16.6 \mathrm{~kg}(15.1 \%)$ and an excessive weight loss of $44.9 \%{ }^{20}$

\section{ENDOSCOPIC SLEEVE GASTROPLASTY}

The endoscopic sleeve gastroplasty procedure is similar to a laparoscopic sleeve gastrectomy in that a portion of the stomach is isolated by creating an uninterrupted series of full-thickness sutures along the length of the stomach ap- proximating the anterior, greater curvature, and posterior walls. An illustration of a continuous stitch configuration is given in Fig. 2. To reduce the endoluminal gastric volume, a cap-based flexible endoscopic suturing system (OverStitch; Apollo Endosurgery, Inc., Austin, TX, USA) is mounted onto a double-channel endoscope to achieve full-thickness. Then, sutures are run through the gastric wall from the antrum to the fundus. ${ }^{21}$

A pilot study was performed to evaluate the feasibility of endoscopic sleeve gastroplasty in 20 patients with a mean age of 45.8 years. The initial body weight $(108.5 \mathrm{~kg})$ was significantly reduced by $8.2 \mathrm{~kg}(7.6 \%), 13.6 \mathrm{~kg}(12.4 \%)$, and 19.3 $\mathrm{kg}(17.8 \%)$, at 1,3 , and 6 months, respectively. ${ }^{22}$

Recent experiences with endoscopic sleeve gastrectomy in 242 patients at three centers demonstrated that the percentage of total body weight loss was $16.8 \%, 18.2 \%$, and $19.8 \%$, at 6 , 12 , and 18 months, respectively. At the 18 -month follow-up, $66.7 \%$ of patients sustained a greater than $15 \%$ total body weight loss. ${ }^{23}$

\section{CONCLUSION}

Flexible endoscopic endoluminal approaches for obesity carry great potential, such as achieving good cosmetic effects via a scarless procedure. They are also safer, more cost-effective, do not require general anesthesia or intubation, require only short-term hospitalization, and involve less reconstruction compared with current surgical approaches. Despite these myriad positive possibilities, the evaluation of 
endoluminal procedures is limited by the small number of clinical studies and the short-term follow-up. In addition, most studies have been performed at only a few centers, with the special devices that are needed for bariatric endoluminal procedures not available in all countries. Further research should be conducted in different regions or countries since obesity rates differ by area. Furthermore, endoscopic gastroplasty should be carefully considered in areas with a high prevalence of Helicobacter pylori infection or gastric cancer due to limitations with respect to gastric cancer screening.

If the more vigorous clinical studies into endoscopic gastroplasty techniques currently in progress prove successful, these techniques are likely to become standard procedures for treating obesity in the near future.

\section{Conflicts of Interest}

The author has no financial conflicts of interest.

\section{REFERENCES}

1. Kim CH, Kim KJ, Kim BY, et al. Prediabetes is not independently associated with microalbuminuria in Korean general population: the Korea national health and nutrition examination survey 2011-2012 (KNHANES V-2,3). Diabetes Res Clin Pract 2014;106:e18-e21.

2. Kannel WB, Cupples LA, Ramaswami R, Stokes J 3rd, Kreger BE, Higgins M. Regional obesity and risk of cardiovascular disease; the Framingham study. J Clin Epidemiol 1991;44:183-190.

3. Mathus-Vliegen EM. Obesity and the gastroenterologist. Endoscopy 2009;41:608-611.

4. Ponce J, Nguyen NT, Hutter M, Sudan R, Morton JM. American society for metabolic and bariatric surgery estimation of bariatric surgery procedures in the United States, 2011-2014. Surg Obes Relat Dis 2015;11:1199-1200.

5. Pandolfino JE, Krishnamoorthy B, Lee TJ. Gastrointestinal complications of obesity surgery. MedGenMed 2004;6:15.

6. Fobi MA. Surgical treatment of obesity: a review. J Natl Med Assoc 2004;96:61-75.

7. Mason EE. Vertical banded gastroplasty for obesity. Arch Surg
1982;117:701-706

8. Laws HL. Standardized gastroplasty orifice. Am J Surg 1981;141:393-394.

9. Chua TY, Mendiola RM. Laparoscopic vertical banded gastroplasty: the Milwaukee experience. Obes Surg 1995;5:77-80.

10. Sugerman HJ, Londrey GL, Kellum JM, et al. Weight loss with vertical banded gastroplasty and Roux-Y gastric bypass for morbid obesity with selective versus random assignment. Am J Surg 1989;157:93-102.

11. Stimac D, Majanović SK. Endoscopic approaches to obesity. Dig Dis 2012;30:187-195.

12. Thompson CC. Endoscopic therapy of obesity: a new paradigm in bariatric care. Gastrointest Endosc 2010;72:505-507.

13. Devière J, Ojeda Valdes G, Cuevas Herrera L, et al. Safety, feasibility and weight loss after transoral gastroplasty: first human multicenter study. Surg Endosc 2008;22:589-598.

14. Familiari P, Boškoski I, Marchese M, Perri V, Costamagna G. Endoscopic treatment of obesity. Expert Rev Gastroenterol Hepatol 2011;5:689701.

15. Moreno C, Closset J, Dugardeyn S, et al. Transoral gastroplasty is safe, feasible, and induces significant weight loss in morbidly obese patients: results of the second human pilot study. Endoscopy 2008;40:406-413.

16. Jafri SM, Arora G, Triadafilopoulos G. What is left of the endoscopic antireflux devices? Curr Opin Gastroenterol 2009;25:352-357.

17. Mahmood Z, McMahon BP, Arfin Q, et al. Endocinch therapy for gastro-oesophageal reflux disease: a one year prospective follow up. Gut 2003;52:34-39.

18. Fogel R, De Fogel J, Bonilla Y, De La Fuente R. Clinical experience of transoral suturing for an endoluminal vertical gastroplasty: 1-year follow-up in 64 patients. Gastrointest Endosc 2008;68:51-58.

19. Brethauer SA, Chand B, Schauer PR, Thompson CC. Transoral gastric volume reduction as intervention for weight management: 12-month follow-up of TRIM trial. Surg Obes Relat Dis 2012;8:296-303.

20. López-Nava G, Bautista-Castaño I, Jimenez A, de Grado T, Fernandez-Corbelle JP. The primary obesity surgery endolumenal (POSE) procedure: one-year patient weight loss and safety outcomes. Surg Obes Relat Dis 2015;11:861-865.

21. Abu Dayyeh BK, Rajan E, Gostout CJ. Endoscopic sleeve gastroplasty: a potential endoscopic alternative to surgical sleeve gastrectomy for treatment of obesity. Gastrointest Endosc 2013;78:530-535.

22. Lopez-Nava G, Galvão MP, da Bautista-Castaño I, Jimenez A, De Grado T, Fernandez-Corbelle JP. Endoscopic sleeve gastroplasty for the treatment of obesity. Endoscopy 2015;47:449-452.

23. Lopez-Nava G, Sharaiha RZ, Neto MG, et al. Endoscopic sleeve gastroplasty for obesity: a multicenter study of 242 patients with 18 months follow-up. Gastroenterology 2016;150(4 Suppl 1):S26. 